\title{
Massive Deep Vein Thrombosis Underlying Unexpected Iliac Vein Compression Syndrome (May-Thurner Syndrome) Complicated by Invasive Electrophysiologic Procedure
}

Ki-Woon Kang, MD, PhD

Cardiology Division, Department of Internal Medicine, Euli University College of Medicine, Deajeon, Korea
Received: June 13, 2015

Revision Received: November 10, 2015

Accepted: December 15, 2015

Correspondence: Ki-Woon Kang, MD

Division of Cardiology, Eulji University Hospital,

Daejeon, Republic of Korea

Tel: +82-42-611-3081, Fax: +82-42-611-3083

E-mail: kwkang@eulji.ac.kr

Copyright (C) 2015 The Official Journal of Korean Heart Rhythm Society Editorial Board \& MMK Co., Ltd.

\begin{abstract}
A 44-year-old woman, who was obese and on oral contraceptive medication, presented with recurrent palpitation to the emergency department. A 12-lead electrocardiography showed narrow QRS complex tachycardia with a short RP interval. On electrophysiology study, typical atrioventricular nodal reentrant tachycardia was confirmed by differential pacing maneuvers, and a radiofrequency slow pathway modification was demonstrated without complication. A week later, the patient complained of pelvic pain and leg swelling. Emergent computer tomography revealed massive deep vein thrombosis (DVT) underlying external iliac vein compression (May-Thurner Syndrome). The case suggests that electrophysiologic procedure with catheterization via the femoro-iliac vein may lead to DVT underlying May-Thurner Syndrome in obese patients on oral contraceptive medication.
\end{abstract}

Key Words: - Deep Vein Thrombosis May-Thurner Syndrome

\section{Introduction}

Recent investigations have found that occurrence of deep vein thrombosis (DVT), complicated by supraventricular tachycardia (SVT) electrophysiology, is extremely rare, with a prevalence of 0

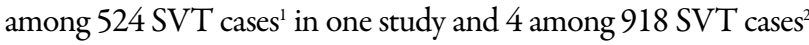
in another. In particular, for young patients, the occurrence of thrombotic events complicated by invasive SVT electrophysiology is rarely observed in clinical practice.

Herein, we report a case of massive DVT complicated by SVT electrophysiology via the femoral approach in a patient with both pre-thrombotic risk (obesity and use of oral contraceptives) and unexpected iliac vein compression syndrome, namely, MayThurner syndrome (MTS).

\section{Case}

A 44-year-woman presented with recurrent palpitation to the emergency department. This patient was obese (body mass index $32 \mathrm{~kg} / \mathrm{m}^{2}$ ), and was on oral contraceptives. Clinically documented electrocardiography (ECG) showed regular, narrow QRS complex tachycardia with a short RP interval and pseudo $r$ in lead 
V1 and pseudo $s$ in lead II and a heart rate of 169 beats per minute (Figure 1A). Conversion to sinus rhythm was seen on ECG after intravenous injection of $6 \mathrm{mg}$ adenosine. A subsequent echocardiogram showed normal cardiac structure and function. A baseline electrophysiology study was planned. Quadripolar and hexapolar electrode catheters (St. Jude Medical, Inc., Minnetonka, MN, USA), inserted via the left femoral vein, were positioned to record the activity of the His bundle and right ventricular (RV) apex. The high right atrium (RA), low RA, and coronary sinus (CS) were mapped with a deflectable duo-decapolar catheter (St. Jude Medical, Inc., St. Paul, MN, USA), which was inserted via the left femoral vein. Intracardiac electrograms were recorded using a Prucka CardioLab ${ }^{\mathrm{TM}}$ electrophysiology system (General Electric Health Care System, Inc., Milwaukee, WI, USA). Baseline intervals were obtained, which were within the normal range. Ventricular burst pacing along with ventricular extrastimulus testing revealed concentric activation and decrement in retrograde activation without a change in activation sequence, likely excluding the possibility of a concealed accessory pathway. Atrial pacing maneuvers and atrial extrastimulus testing were utilized to assess for the presence of two functional pathways, also known as dual AV nodal physiology. Atrial burst pacing resulted in the induction of a sustained narrow complex tachycardia with a cycle length (CL) of $348 \mathrm{msec}$. While in clinical tachycardia, VA conduction appeared almost simultaneous with a VA time of $40 \mathrm{msec}$, maintaining an atrialHis/His-atrial ratio (AH/HA) $>1$, highly suggestive of typical atrioventricular nodal reentrant tachycardia (Figure 1B). Entrainment of this tachycardia demonstrated a V-A-V response.

Based on the evidence, radiofrequency ablation (RF) was performed at a slow pathway region using a 7-Fr, 4-mm-tip deflectable ablation catheter (Boston Scientific Corp., Natick, MA, USA), via the right femoral vein. With the application of RF energy, accelerated junctional rhythm was recorded, which corresponds highly to successful ablation of slow pathway conduction. No further tachycardia was induced, despite isoproterenol infusion. No complication was found after the procedure.

One week later, she visited in out-patient clinic with pelvic pain and progressive swelling in the left leg. The left leg of the patient was slightly pale but with palpable pulses. Emergent abdominal computed tomography (CT) showed abnormally sluggish blood flow in the proximal deep venous system, which confirmed multiple large occlusive thrombi in the deep venous system of her left leg, extending from the level of the femoral vein to the inferior vena cava (IVC) underlying MTS (Figure 2). The patient was hospitalized for further evaluation and treatment of massive DVT. Coagulopathy-related blood tests revealed negative results. During intravenous infusion of unfractionated heparin, she developed sudden chest discomfort. A subsequent chest CT showed multiple fragmented thrombi in her left pulmonary trunk. However, there was no evidence of RV dysfunction on echocardiography (Figure 3A). Multiple, large occlusive thrombi in the left femoral and iliac vein were still not resolved (Figure 3B). Therefore, prophylactic IVC filter was applied before attempting thrombolysis and intravascular catheter-based local thrombolysis therapy, which resulted in complete resolution of the thrombus (Figure 4). Finally, IVC filter was removed with the hook at the lower aspect of the filter through a transfemoral approach.

\section{Discussion}

MTS, or iliac vein compression syndrome, is an anatomic variant where the left iliac vein is compressed by the right iliac artery. Arterial pulsation causes venous intimal proliferation, forming loose connective tissue, "spurs", which cause turbulent flow and contribute to venous thrombosis. DVT is thought to be the initial presenting symptom in patients with MTS. This includes patients with left lower extremity swelling, pain, discoloration, and/or skin ulcers. The exact incidence and prevalence of MTS are still unknown. However, it has been estimated to occur in $2-5 \%$ of patients with lower extremity venous disorder. Some studies have suggested that MTS exists in as many as $22-24 \%$ of these patients. Most MTS cases are undiagnosed in asymptomatic patients, because of collateral drainage of the left lower extremity through the internal iliac veins and the left gonadal vein. In some cases, MTS have been diagnosed as venous hypertension and insufficiency, recurring thrombosis, post-thrombotic syndrome, iliac vein rupture, or pulmonary embolism. ${ }^{3.4}$

In this case report, we describe an obese patient on oral contraceptives with unexpected underlying MTS that contributed 

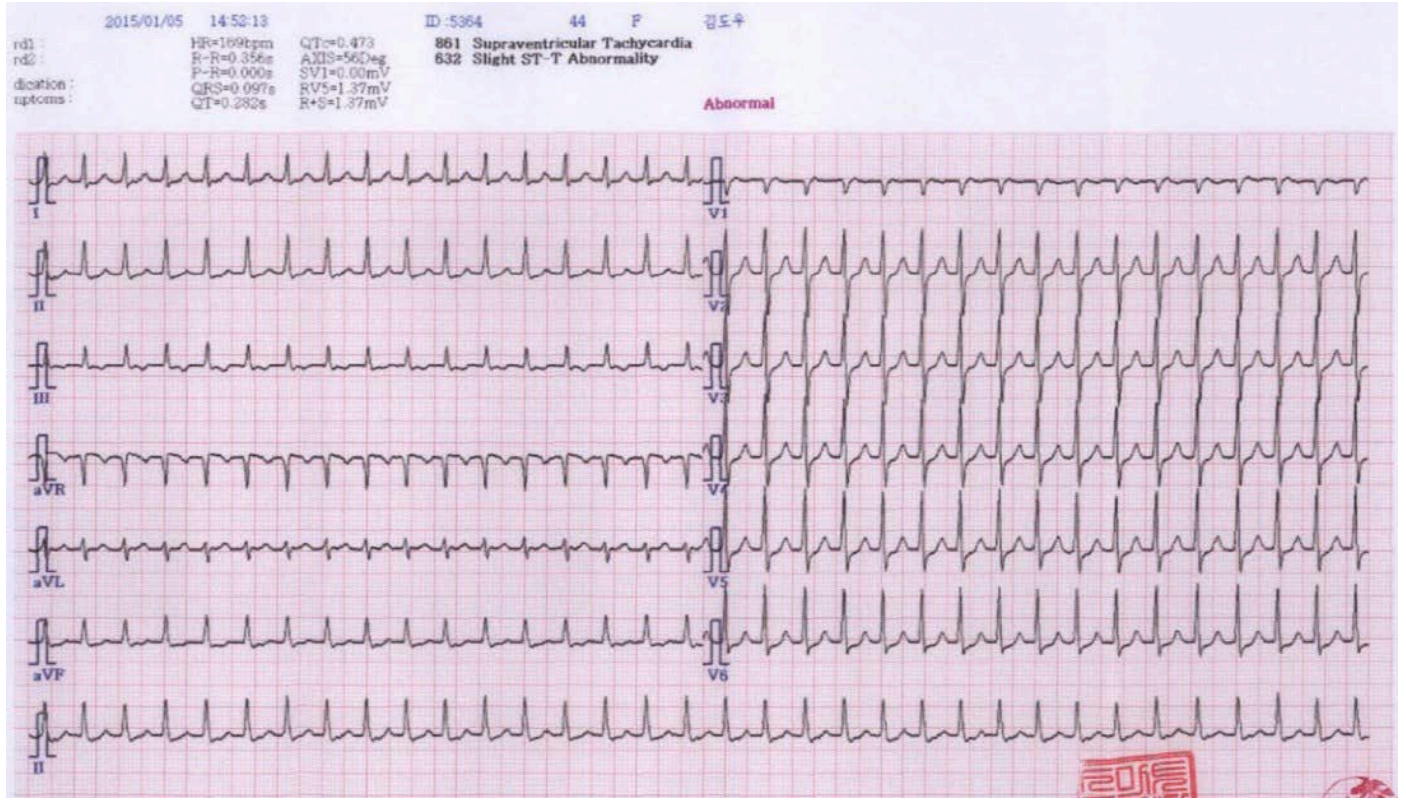

B

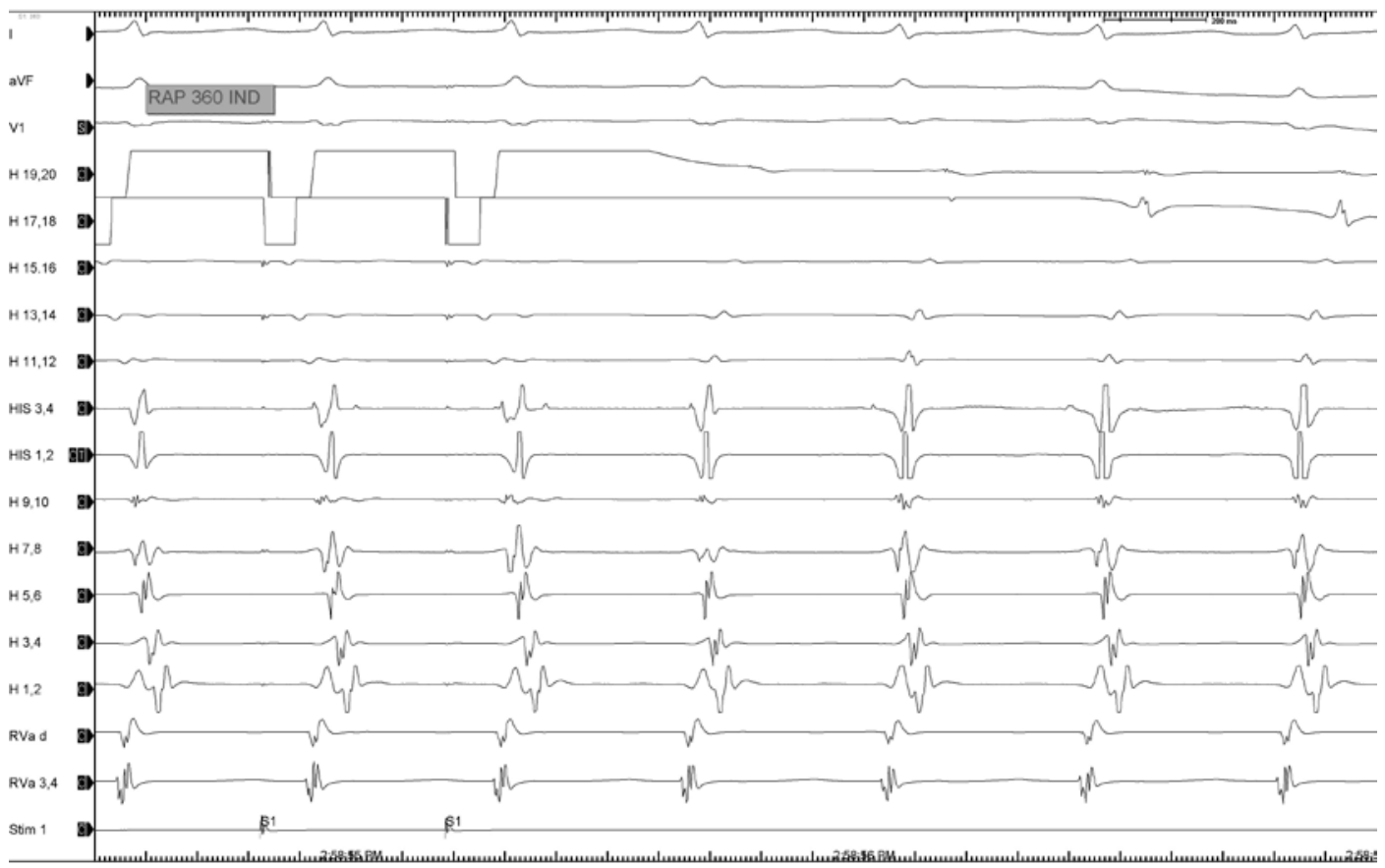

Figure 1. (A) 12-lead electrocardiography in the emergency department showing narrow QRS complex tachycardia. (B) Intracardiac electrograms showing induction of atrioventricular nodal reentrant tachycardia. 
A

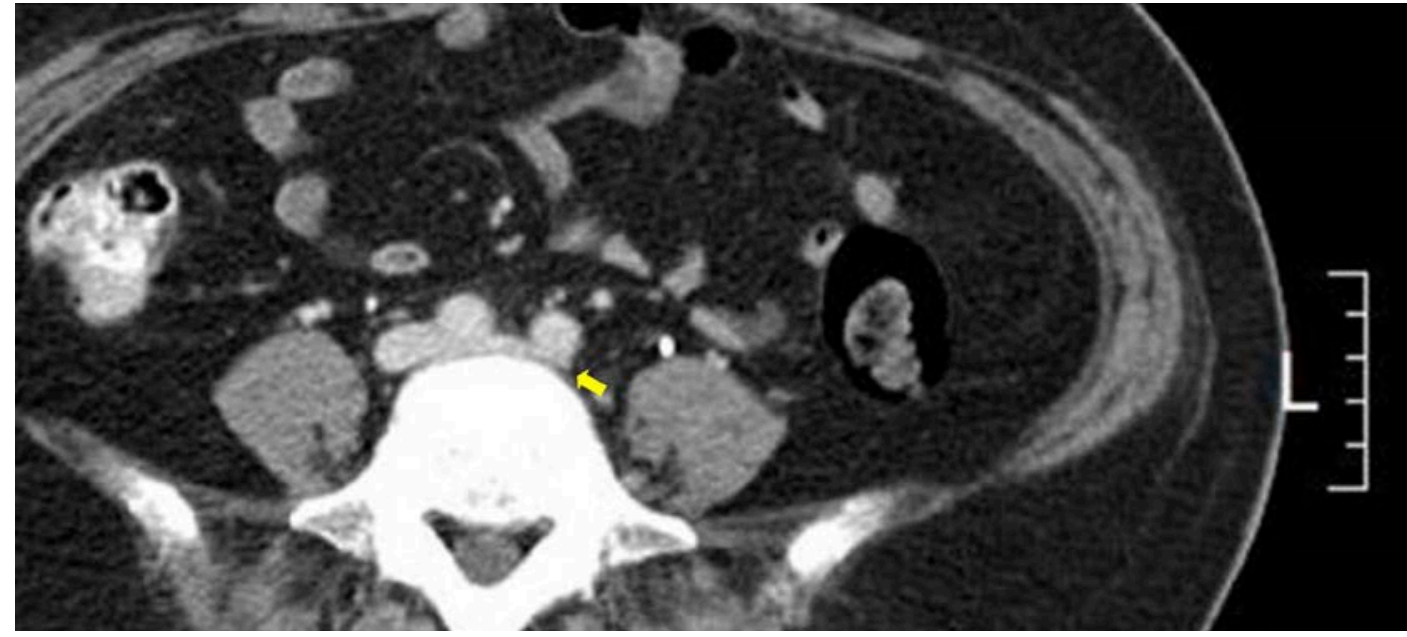

B
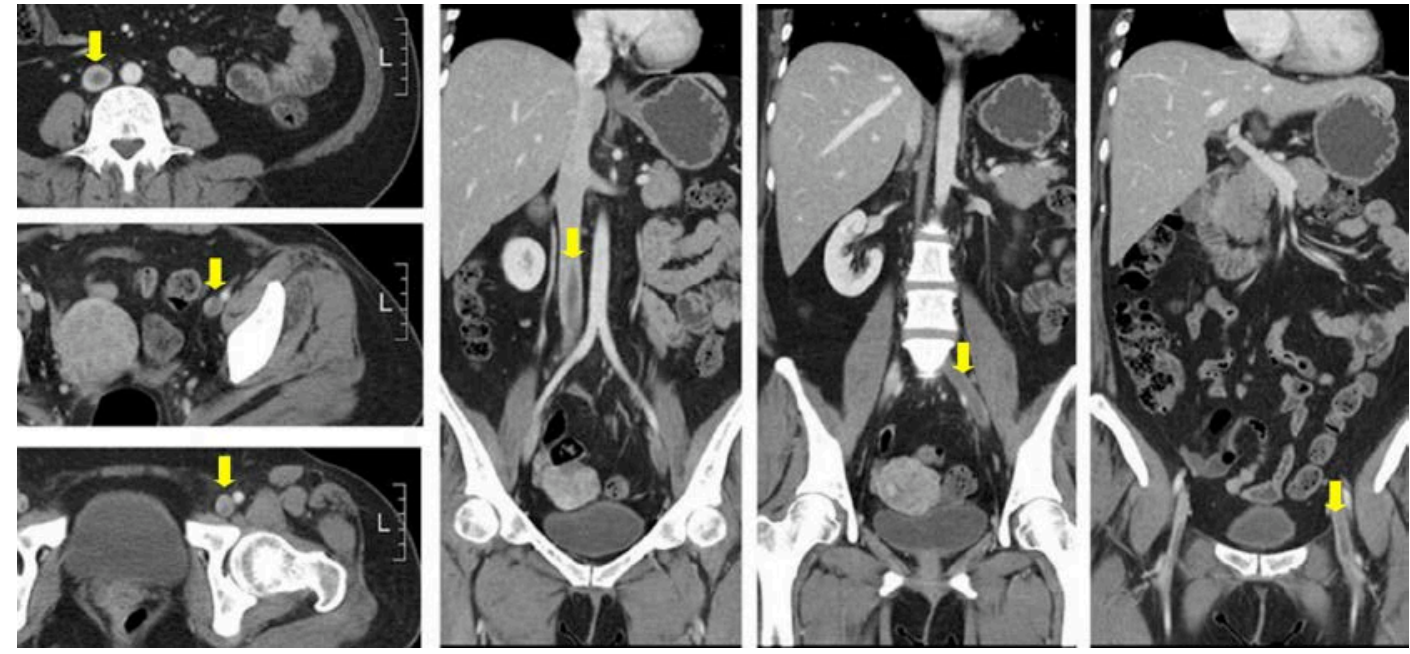

Figure 2. (A) Abdomen computed tomography (CT) showing the left common iliac vein just posterior to the right common iliac artery. Yellow arrow indicates that compression of the left common iliac vein between the right common iliac artery and the lumbosacral spine is the basis of a diagnosis for iliac compression syndrome (May-Thurner syndrome). (B) Yellow arrows indicating multiple filling defects of the left iliac-femoral vein and inferior vena cava.

to the development of extensive massive DVT. The invasive SVT electrophysiologic procedure was a key precipitating factor for massive DVT because of of a decrease in ambulatory status and left lower extremity venous drainage compromise.

Contrast venography is the gold standard for diagnosis and for determining the therapeutic strategy, because color duplex ultrasound cannot identify the anatomical defect of MTS. Contrast-enhanced CT of the abdomen and magnetic resonance venography can also be used for diagnosis based on anatomic changes or distribution of thrombus but their use is limited when thrombosis obscures the underlying anatomic abnormality. Anticoagulation alone may not be adequate because of the large- volume of clot burden and the lack of treatment of the underlying venous stenotic lesion. The main treatment for MTS-associated DVT is thrombectomy or thrombolysis. ${ }^{4}$

Patients with isolated unilateral left lower extremity DVT terminating at the iliac vein confluence should be examined for MTS, particularly in the invasive post-procedure period. In this instance, prompt and adequate imaging-based diagnosis of both massive DVT and underlying MTS is required. Local thrombolysis with an IVC filter and not anticoagulation can relieve symptoms and prevent catastrophic consequences, including massive pulmonary thromboembolism and sudden death. 
A


B
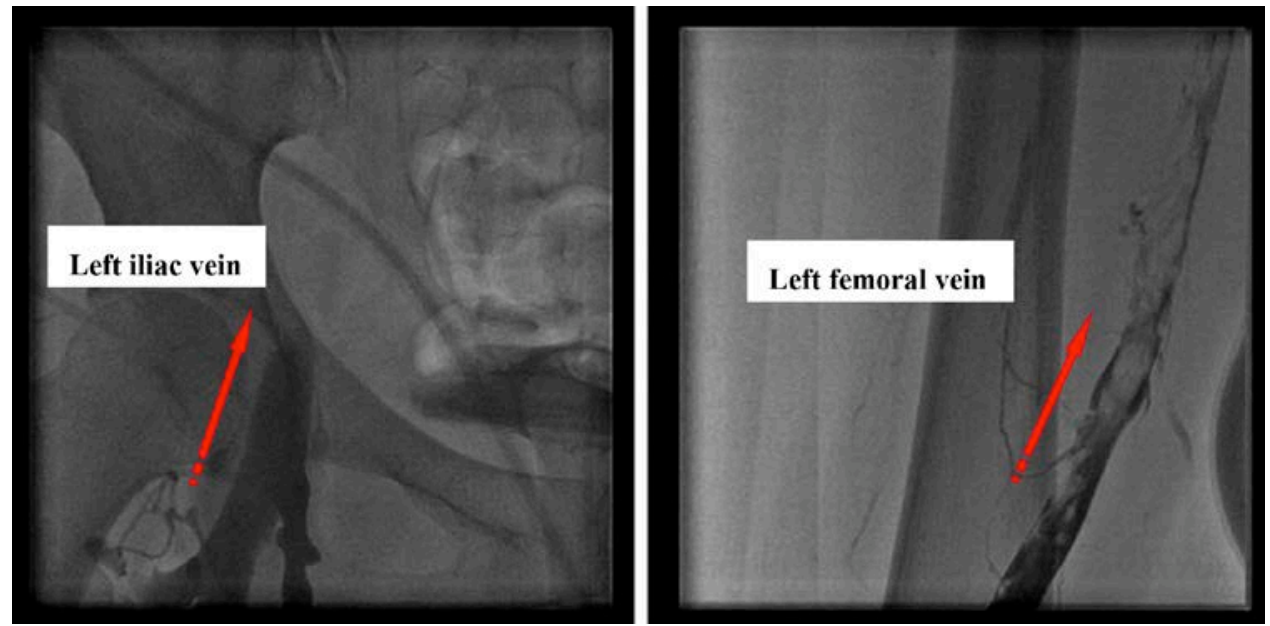

Figure 3. (A) Yellow arrows indicating multiple fragmented thrombi in the left pulmonary trunk during anticoagulation. However, there was no evidence of right ventricular dysfunction on echocardiography. (B) In the prone position, venogram showing an occlusive thrombus in the left common iliac vein (left) and large thrombus burden in the left femoral vein (right) with red arrow indicating venous flow.



Figure 4. Yellow arrow indicating complete resolution of thrombus and restoration of flow in the common iliac vein in the prone position after thrombolysis with an inferior vena cava (IVC) filter with red arrow indicating venous flow. 


\section{References}

1) Hussain SK, Eddy MM, Moorman L, Malhotra R, Darby AE, Bilchick K, Mason P, Mangrum MJ, Dimarco JP, Ferguson JD. Major complications and mortality within 30 days of an electrophysiological procedure at an academic medical center: implications for developing national standards. J Cardiovasc Electrophysiol. 2015;26:527-531.

2) Bohnen M, Stevenson WG, Tedrow UB, Michaud GF, John RM, Epstein LM, Albert CM, Koplan BA. Incidence and predictors of major complications from contemporary catheter ablation to treat cardiac arrhythmias. Heart Rhythm. 2011;8:1661-1666.

3) May R, Thurner J. The cause of the predominantly sinistral occurrence of thrombosis of the pelvic veins. Angiology. 1957;8:419-427.

4) Mousa AY, AbuRahma AF. May-Thurner syndrome: update and review. Ann Vasc Surg. 2013;27:984-995. 